\title{
Draft genome of Thermomonospora sp. CIT 1 (Thermomonosporaceae) and in silico evidence of its functional role in filter cake biomass deconstruction
}

Wellington P. Omori ${ }^{1}$, Daniel G. Pinheiro ${ }^{2}$, Luciano T. Kishi ${ }^{3}$, Camila C. Fernandes ${ }^{3}$, Gabriela C. Fernandes ${ }^{1}$, Elisângela S. Gomes-Pepe ${ }^{3}$, Claudio D. Pavani ${ }^{1}$, Eliana G. de M. Lemos ${ }^{3}$ and Jackson A. M. de Souza $^{4}$ iD

${ }^{1}$ Programa de Pós-Graduação em Microbiologia Agropecuária, Faculdade de Ciências Agrárias e Veterinárias, Universidade Estadual Paulista (UNESP), Jaboticabal, SP, Brazil.

${ }^{2}$ Departamento de Tecnologia, Laboratório de Bioinformática, Faculdade de Ciências Agrárias e

Veterinárias, Universidade Estadual Paulista (UNESP), Jaboticabal, SP, Brazil.

${ }^{3}$ Laboratório Multiusuário Centralizado para Sequenciamento de DNA em Larga Escala e Análise de Expressão Gênica (LMSeq), Faculdade de Ciências Agrárias e Veterinárias, Universidade Estadual Paulista (UNESP), Jaboticabal, SP, Brazil.

${ }^{4}$ Departamento de Biologia Aplicada à Agropecuária, Laboratório de Genética Aplicada, Faculdade de Ciências Agrárias e Veterinárias, Universidade Estadual Paulista (UNESP), Jaboticabal, SP, Brazil.

\begin{abstract}
The filter cake from sugar cane processing is rich in organic matter and nutrients, which favors the proliferation of microorganisms with potential to deconstruct plant biomass. From the metagenomic data of this material, we assembled a draft genome that was phylogenetically related to Thermomonospora curvata DSM 43183, which shows the functional and ecological importance of this bacterium in the filter cake. Thermomonospora is a gram-positive bacterium that produces cellulases in compost, and it can survive temperatures of $60^{\circ} \mathrm{C}$. We identified a complete set of biomass depolymerizing enzymes in the draft genome of Thermomonospora sp. CIT 1 , such as $\alpha$-amylase, catalase-peroxidases, $\beta$-mannanase, and arabinanase, demonstrating the potential of this bacterium to deconstruct the components of starch, lignin, and hemicellulose. In addition, the draft genome of Thermomonospora sp. CIT 1 contains 18 genes that do not share identity with five other species of Thermomonospora, suggesting that this bacterium has different genetic characteristics than those present in genomes reported so far for this genus. These findings add a new dimension to the current understanding of the functional profile of this microorganism that inhabits agro-industrial waste, which may boost new gene discoveries and be of importance for application in the production of bioethanol.
\end{abstract}

Keywords: Actinobacteria, pectin, hemicellulose, crystalline cellulose, orthologous genes.

Received: December 14, 2017; Accepted: June 10, 2018.

A by-product of sugar manufacture, the filter cake is very rich in organic matter, phosphorus, moisture (70$80 \%$ ), and other nutrients. The filter cake is a mixture of the decantation sludge with residual particles of sugarcane bagasse that results from the process of milling and extraction of the cane broth. Produced in large quantities, the filter cake can be used in natura as a substitute for commercial soil fertilizers (de Fravet et al., 2010), or it can be stored in an appropriate place for up to 40 days before use in agricul-

Send correspondence to Jackson A. M. de Souza. Department of Biology Applied to Agricultural and Livestock, School of Agricultural and Veterinary Sciences, São Paulo State University (UNESP), Via de Acesso Prof. Paulo Donato Castellane Km 05, 14884-900, Jaboticabal, SP, Brazil. E-mail: jackson@fcav.unesp.br. ture, making it an environmental conductive agent for the development of numerous microorganisms.

Wastes from agriculture and plant biomass composting are natural habitats of Thermomonospora curvata (Thermomonosporaceae) bacteria (Wang et al., 2016) that can survive temperatures of $60{ }^{\circ} \mathrm{C}$ or above (Wei et al., 2014). Actinobacteria have a slow growth rate and one of the most abundant microbial groups in composts, a fact related mainly to adaptive advantages related to lignocellulases production capacity and secretion of antimicrobial agents (Martins et al., 2013; Wang et al., 2016). However, these lignocellulases must be able to overcome the recalcitrance of plant biomass, allowing the release of sugars (pentoses and hexoses) and nutrients, which can be used in biological processes fundamental to cell maintenance. The 
recalcitrance of lignocellulose occurs due to cellulose paracrystallinity, molecular complexity of hemicellulose coating on cellulose microfibrils, and the interpenetration and encapsulation of polysaccharide components by lignin (Cragg et al., 2015).

Enzymes capable of deconstructing lignocellulose are widely used in the pretreatment of plant biomass for the production of bioethanol, where the sugars derived from this process can be used in the fermentation. In fact, the use of enzymes in this type of process is the most indicated from the environmental and economic point of view, since, to overcome the recalcitrance of the plant biomass, it is necessary to perform severe chemical and/or physical pretreatments (Klein-Marcuschamer et al., 2012; Cragg et al., 2015). This results in the production of undesirable byproducts such as release of effluents of high toxicity to the environment that must be neutralized, which raises production costs. However, for the production of bioethanol to be implemented on a large scale and in a competitive manner, it is essential to reduce the cost of enzyme production and have an effective production schedule cocktail (in mg per ton) as well as the optimization of the enzymatic composition to increase efficiency through the discovery and kinetic characterization of new enzymes (KleinMarcuschamer et al., 2012).

Thermomonospora curvata (Thermomonosporaceae) has a genetic arsenal with great biotechnological potential (Wei et al., 2014; Wang et al., 2016). However, many studies did not adequately explore other characteristics of the genome of this microorganism, such as identification of genes related to lignocellulose degradation. In this context, materials rich in plant matter such as agro-industrial waste deposits favor the selection of genes with potential biomass deconstruction, creating a favorable environment for the discovery of new strains with new enzymes or unpublished genes. In this work, we report genes present in the draft genome CIT 1 that is phylogenetically related to $T$. curvata DSM 43183, which was reconstructed from sequences derived from total metagenome of filter cake.

In January 2013, two random samples were collected at $0-20 \mathrm{~cm}(393 \mathrm{~mL})$ depth of the surface of the filter cake stack and stored for approximately 40 days. The location of the collection was Fazenda Itaquerê in Nova Europa, state of São Paulo, Brazil. Samples were transported immediately to the laboratory and after homogenization and formation of a composite, the sample was weighed to $250 \mathrm{mg}$ of total metagenomic DNA extraction using Fast DNA ${ }^{\mathbb{B}}$ kit for Soil (Bio 10, Quantum Biotechnologies), following the manufacturer's instructions. The purity and concentration of the DNA were analyzed in Qubit $^{\circledR} 2.0$ Fluorometer (Life Technologies) with the Qubit dsDNA BR Assay Kit (Invi$\operatorname{trogen}^{\circledR}$ ), following the manufacturer's recommendations. A sample of the filter cake was sent for bromatological analysis.
The metagenomic DNA sequencing library was prepared according to the TruSeq ${ }^{\circledR}$ DNA Sample Preparation v2 $\left(\right.$ Illumina ${ }^{\circledR}$ ) protocol, as per the manufacturer's recommendations. Sequencing of the shotgun library ( $1 \mu \mathrm{g}$ DNA) was performed on the Illumina ${ }^{B}$ platform, HiScanSQ equipment, using Paired-End Cluster Generation Kit v3 (Illumina ${ }^{\circledR}$ ) and TruSeq SBS Kit v3 - 200 Cycles (Illumina $^{\circledR}$ ), following the recommendations of the manufacturer.

Removal of sequences with low quality $(\mathrm{Q}<20)$ and length less than $50 \mathrm{bp}$, as well as assembly of scaffolds, formation of genome clusters recovered from metagenomic data, closure of gaps to increase scaffold extension and verification of coverage sequencing were performed as previously described (Jourda et al., 2015; Kishi et al., 2015). At the end, 29 scaffolds were retrieved, which were annotated automatically on the online RAST server (Aziz et al., 2008). The prediction of carbohydrase enzymes was done using the dbCAN server (database Carbohydrate-active enzyme ANnotation) (Yin et al., 2012). In order to verify if the genes predicted by dbCAN were similar (best hits, $\geq$ $85 \%$ of coverage) to some carbohydrase gene deposited in GenBank, we performed a blastp search (Altschul et al., 1990) against the non-redundant protein database of the National Center for Biotechnology Information (NCBI).

After searching the housekeeping genes of 19 bacterial species phylogenetically related to the draft genome CIT 1, the sequences were aligned separately using Mafft v.7.215 (Katoh and Standley, 2013) and subsequently concatenated with Mesquite v.2.74 (Maddison and Maddison, 2017). The prediction of the best nucleotide substitution model, based on the lower Akaike Information Criterion correction (AICc) value, was performed with the online program IQ-TREE v.1.5.5 (Trifinopoulos et al., 2016). For the construction of the phylogenetic tree, we used the IQTREE program by selecting the nucleotide substitution matrix with the lowest AIC, correction option for frequency states optimized for Maximum Likelihood (ML) and bootstrap with 1,000 replicates using the algorithm Ultrafast (Trifinopoulos et al., 2016).

We used the online program OrthoVenn (Wang et al., 2015 ) to estimate the orthologous genes and distinguish between the singularities of draft genomes of CIT 1 and Thermomonospora curvata DSM 43183 (Thermomonosporaceae, accession CP001738). To verify the proximity between genomes, we used the online software JspeciesWS v.3.0.11 (Richter et al., 2016), which uses pairwise alignment between genome sequences to determine the percentage of similarity between organisms from the average nucleotide identity (ANI) parameters based on blast (ANIb), MUMmer (ANIm), and tetranucleotide signature correlation index (Tetra).

This Whole Genome Shotgun project has been deposited at DDBJ/ENA/GenBank under the accession MOYN00000000. The version described in this paper is 
MOYN02000000. In RAST server, the accession number is 471852.20 .

After separating the genome groups of the metagenomic data of the filter cake, 101 scaffolds that were not similar ( $\leq 70 \%$ and coverage $\leq 80 \%)$ were identified and eliminated from the draft genome CIT 1 dataset of Thermomonospora curvata (Thermomonosporaceae) (Table S1). According to RAST platform, the draft genome CIT 1 presented a size of $5,460,082 \mathrm{bp}$, GC content of $71.8 \%$ and 4,834 coding sequences (CDS), data very similar to those observed in the circular curve genome of $T$. curvata DSM 43183 (Table S2). The total sequencing showed 49,554,712 paired-end reads, from where the draft genome was assembled from $9.48 \%$ of the reads, with $180 X$ coverage. According to data from this same platform, the enzymes involved in carbohydrate metabolism were distributed among the central metabolism subsystems of carbohydrates (146), fermentation (73), polysaccharides (28), and monosaccharides (24), demonstrating the potential of using sugars derived from the plant fiber present in the filter cake.

The analysis in dbCAN for draft genome CIT 1 identified 170 candidate genes of the carbohydrases synthesis, whereas the same analysis performed on the Thermomonospora curvata DSM 43183 (Thermomonosporaceae) showed only 93 genes of the same group (Tables S3 and S4). Among these, genes of major interest for bioethanol production and little explored in the genome of Thermomonospora species are those related to the classes of glycoside hydrolases (GH) $\beta$-mannanase (GH26), arabinanase (GH43) e $\alpha$-amylase (GH13) (Table S3). These enzymes act on various lignocellulosic structures (Figure 1A-D), and such structures were identified in the bromatological analysis of the filter cake in the proportions of $25 \%$ cellulose, $9 \%$ lignin, $8 \%$ hemicellulose, and $8 \%$ non-nitrogenated extract (pectin, starch, etc.).

The 29 scaffolds of the draft genome CIT 1 were very similar (mean of 99\%) to the circular genome of Thermomonospora curvata DSM 43183 (Thermomonosporaceae). Phylogenetic analysis allowed confirming the taxonomic positioning of the draft genome CIT 1 (100\% bootstrap) as belonging to the species of T. curvata (Figure 2A). This classification is consistent with the high similarity $(>99 \%$ ) between pairs indicated by the $\%$ ANIb, $\%$ ANIm and, Tetra indexes (Richter et al., 2016) obtained with JSpeciesWS (Table 1). However, the analysis performed with OrthoVenn, showed that of the 3,756 clusters of orthologous genes present in the draft genome of Thermomonospora sp. CIT 1, only 18 clusters are not shared with any other species (Figure 2B and C). According to Gene Ontology (GO), these genes are mainly related to the function with hydrolases activity, acting on $\mathrm{C}-\mathrm{O}, \mathrm{C}-\mathrm{N}$ and, C-C bonds (GO:0016787), peptidase activity (GO:0008233) and, genes related to the transport of macro-

\begin{tabular}{|l|l|}
\hline \multicolumn{1}{|c|}{ CARBOHYDRATES } \\
\hline$\square$ L-Rhamnose \\
$\bigcirc$ & Glucuronic acid \\
$\diamond$ & L-Arabinose \\
$\square$ & D-Galactose \\
$\nabla$ & Felic acid \\
$\nabla$ & Acetyl \\
$\square$ & D-Mannose \\
$\bigcirc$ & D-Glucose \\
$\square$ & D-Glucose oxidized \\
\hline
\end{tabular}

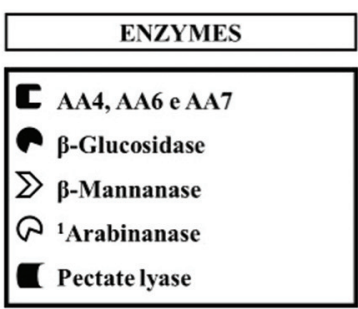

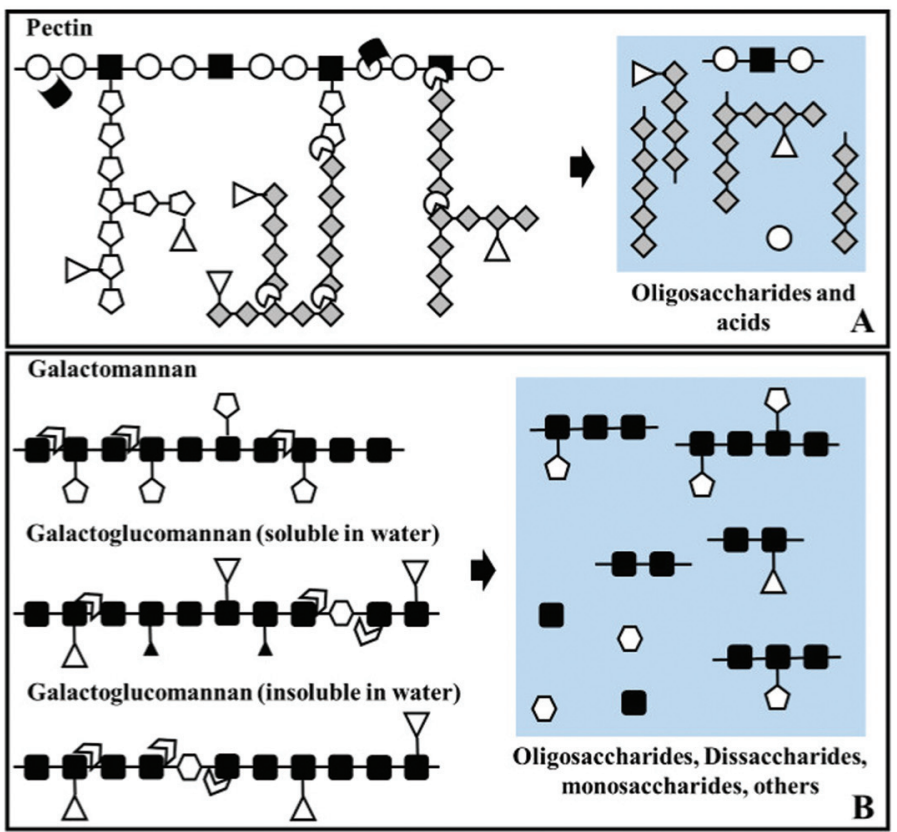

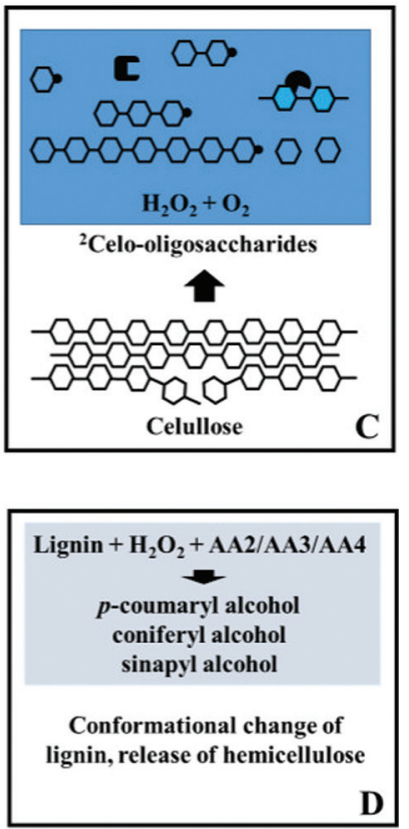

Figure 1 - Identification of genes that can act on the main plant biomass structures in the filter cake present in the draft genome CIT 1. (A) Enzymes that act on pectin, releasing mainly glucuronic acid and polysaccharides. (B) Enzymes that act on the main constituents of hemicellulose, releasing oligosaccharides, disaccharides, monosaccharides, etc.. (C) Enzymes that can act on the cellulose, releasing components of cello-oligosaccharides and glucose. (D) mechanism of action of some Auxiliary Activities (AA) that act on the conformational change of lignin and availability of structural components with aromatic rings of this heteropolymer. ${ }^{1}$ According to the database BRENDA (https://www.brenda-enzymes.org/), acts best on linear 1,5- $\alpha$-L-arabinan. Also acts on branched arabinan, but more slowly. ${ }^{2}$ In addition to cellobiose, AAs can oxidize other cello-oligosaccharides, such as hepta-cellobiose, trio-cellobiose, etc. 

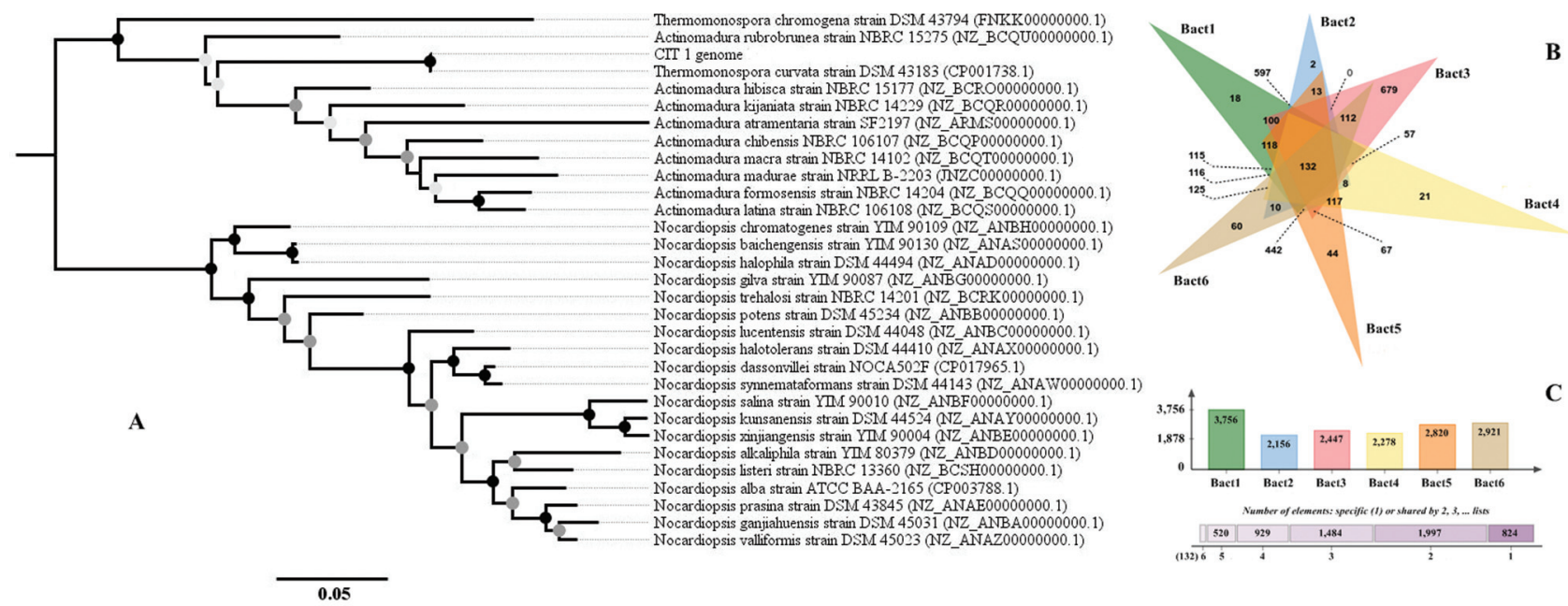

Figure 2 - Phylogenetic analysis using Maximum Likelihood to verify the phylogenetic position of the draft genome CIT 1 (Thermomonospora sp. CIT 1) and identification of clusters of orthologous genes using Ortho Venn. (A) Phylogenetic tree demonstrating that the draft genome CIT 1 is related to the clade of Thermomonospora curvata DSM 43183 (Thermomonosporaceae). (B) Clusters of orthologous genes shared among the six phylogenetically related species that make up the Thermomonosporaceae family. The draft genome of Thermomonospora sp. CIT 1 (Bact1) shares 597 clusters of orthologous genes with T. curvata DSM 43183 (Bact2), possessing 18 clusters that are only present in the draft genome of Thermomonospora sp. CIT 1. (C) Total clusters of orthologous genes verified in the six species analyzed, demonstrating that the six bacteria share a total of 132 clusters of orthologous genes among themselves. We retrieved from GenBank the nucleotide sequences of the $23 \mathrm{SrRNA}$, recA, dnaK, dnaJ, dnaJ, atpD and, rpoB genes from 19 species of Nocardiopsis, nine species of Actinomadura, and two species of Termomonospora. The circles found at the Maximum Likelihood phylogenetic tree nodes represent the bootstrap support value obtained for each branch. Filled black circles represent bootstrap between 90-100\%; filled dark gray circles represent bootstrap between 70-89\%; filled light gray circles represent bootstrap between 50-69\%. Bact1: draft genoma CIT 1; Bact2: Thermomonospora curvata strain DSM 43183 (Thermomonosporaceae); Bact3: Actinomadura rubrobrunea strain NBRC 15275; Bact4: Thermomonospora chromogena strain DSM 43794; Bact5: Actinomadura hibisca strain NBRC 15177; Bact6: Actinomadura kijaniata strain NBRC 14229

Table 1 - Probability pairwise comparison between the circular genome Thermomonospora curvata DSM 43183 and other 14 genomes of Actinobacteria.

\begin{tabular}{|c|c|c|c|c|c|c|}
\hline Strain & Genome (bp) & $\% \mathrm{GC}$ & Gene & $\% \mathrm{ANIb}$ & $\%$ ANIm & $\%$ Tetra \\
\hline${ }^{1}$ genome CIT 1 & $5,460,082$ & 71.80 & 4,906 & $99.45(90.79)^{2}$ & $99.62(95.28)$ & 99.99 \\
\hline Thermomonospora chromogena DSM 43794 & $5,988,017$ & 71.00 & 5,122 & $73.15(27.17)$ & $83.75(8.20)$ & 95.57 \\
\hline Actinomadura atramentaria DSM 43919 & $6,709,087$ & 73.70 & 6,288 & $75.19(37.30)$ & $84.27(14.23)$ & 87.08 \\
\hline Actinomadura chibensis NBRC 106107 & $9,408,892$ & 72.80 & 8,425 & $75.64(41.77)$ & $84.07(16.18)$ & 88.19 \\
\hline Actinomadura formosensis NBRC 14204 & $7,079,728$ & 71.60 & 6,658 & $76.02(40.35)$ & $84.29(17.30)$ & 92.31 \\
\hline Actinomadura hibisca NBRC 15177 & $9,023,974$ & 72.40 & 8,470 & $76.78(42.49)$ & $84.65(23.49)$ & 94.30 \\
\hline Actinomadura kijaniata NBRC 14229 & $10,258,935$ & 73.20 & 9,846 & $76.91(41.05)$ & $84.57(22.93)$ & 95.17 \\
\hline Actinomadura latina NBRC 106108 & $7,867,648$ & 72.00 & 7,275 & $75.98(42.07)$ & $84.07(18.27)$ & 92.05 \\
\hline Actinomadura macra NBRC 14102 & $9,046,351$ & 70.70 & 8,032 & $75.46(39.83)$ & $84.18(15.60)$ & 92.24 \\
\hline Actinomadura rifamycini DSM 43936 & $8,385,217$ & 74.10 & 7,628 & 75.88 (41.49) & $84.25(16.94)$ & 89.40 \\
\hline Actinomadura rubrobrunea NBRC 15275 & $6,724,167$ & 72.80 & 5,901 & $77.62(39.24)$ & $85.12(23.98)$ & 95.12 \\
\hline Nocardiopsis alba ATCC BAA-2165 & $5,848,211$ & 69.70 & 5,103 & $71.09(21.50)$ & $83.4(4.11)$ & 87.35 \\
\hline Nocardiopsis alkaliphila YIM 80379 & $5,209,552$ & 67.50 & 4,722 & $70.78(19.88)$ & $83.14(3.13)$ & 81.97 \\
\hline Nocardiopsis baichengensis YIM 90130 & $6,404,110$ & 73.60 & 5,580 & $72.22(25.47)$ & $83.37(7.41)$ & 94.18 \\
\hline
\end{tabular}

${ }^{1}$ Partial genome retrieved from metagenomic DNA sequencing data from filter cake stored for 40 days.

2Percentage of total bases of the analyzed genome that aligned against the genome of T. curvata DSM 43183.

molecules, small molecules, and ions through the cell wall (GO:0005215) (data not shown). Clusters related to transport of sugars through the membrane were also corroborated by Uniprot data (also via Ortho Venn) showing their importance to bioethanol production by biotechnology exploitation.

The membrane transporter cluster has many unknown functions, but one of its most prominent activities is to 
transport molecules of hexoses (D-mannose and D-glucose) and pentoses (L-arabinose) (Beisel and Afroz, 2016). Thus, these characteristics demonstrate that these monosaccharide molecules are derived from the deconstruction of pectin, hemicellulose, and cellulose (Vries and Visser, 2001), which may denote certain specialization of these bacteria in deconstructing (Figure 1) and capturing these molecules to use them in their metabolism.

With almost double the number of genes related to lignocellulose depolymerization in relation to the genome of Thermomonospora curvata DSM 43183 (Thermomonosporaceae) (Tables S3 and S4), the draft genome CIT 1 presented genes that can act on pectin and many structures of lignocellulose (Figure 1A-D). Among the specific genes of the draft genome CIT 1 that have catalytic activity on lignin, hemicellulose (galactomannan and galactoglucomannan), and starch structures are the peroxidase, arabinanase (Figures 1B and 1D), and $\alpha$-amylase (Table S3). Representing peroxidases, AAs often act in synergy with the GHs during the phases of depolymerization of lignocellulosic materials, acting on lignin and producing a set of heterogeneous aromatic compounds, which are capable of being metabolized by the microorganisms (Cragg et al., 2015 ). The $\alpha$-amylases have as specific substrate, the polysaccharide starch, providing small units of glucose and maltose (Gopinath et al., 2017).

The identification of many genes and metabolic pathways related to depolymerization of plant biomass, together with the identification of remaining lignocellulosic material in the filter cake, suggest that the draft genome CIT 1 has a complete set of mechanisms that can act on the deconstruction of carbohydrate polymers (Table S3 and Figure 1A-D). These mechanisms favor the release of sugars (monosaccharides, disaccharides, and oligosaccharides) that can be used as a source of energy (Vries and Visser, 2001).

Phylogenetic analysis showed that the draft genome CIT 1 clusters in the clade of Thermomonospora curvata DSM 43183 (Thermomonosporaceae) (Figure 2A). This result was supported by analyses of orthologous genes with Ortho Venn (Figure 2B) and ANI and Tetra nucleotide of JspeciesWS (Table 1). In addition, this taxonomic classification is in line with what was expected for this bacterial family (Kroppenstedt and Goodfellow, 2006). We observed the formation of a monophyletic pair between the draft genome CIT 1 and T. curvata DSM 43183, whereas there is formation of paraphyletic groups in the branches representing the species of Actinomadura and between $T$. chromogena and A. rubrobrunea (Figure 2A). This difficulty in increasing the resolution of the phylogenetic classification is related to the lack of data available in the public databases, since the species of $T$. curvata have only one complete genome and two partial genomes published in GenBank so far (November 2017, including the draft genome Thermomonospora sp. CIT 1 of this study).
The storage of the filter cake for long periods causes changes in its characteristics, such as loss of moisture and nutrients. In part, these modifications are due to the microbial activity in the natural substrate. The consumption of nutrients gives rise to a recalcitrant environment that increases the microbial competition and favors the occurrence of horizontal gene transfer. This mechanism may have conferred adaptive advantages for the microbial species under study from CIT 1.

The results of the predictions discussed here have not yet been extensively explored in the genomes of Thermomonospora species published to date. Our findings are relevant to direct research involving the production of bioethanol since the analysis of the draft genome of Thermomonospora sp. CIT 1 (Thermomonosporaceae) extracted from the filter cake revealed that this bacterium has almost double the number of genes that participate in the release of disaccharides and monosaccharides in relation to the genome of T. curvata DSM 43183. These genes can act on the main constituents of hemicellulose (galactomannan and galactoglucomannan), lignin, cellulose, starch, and pectin.

\section{Acknowledgments}

We thank the Agricultural Microbiology Program for the academic support and Coordenação de Aperfeiçoamento de Pessoal de Nível Superior - Brasil (CAPES) - Finance Code 001 for the financial support granted to the graduate student. Financial support for the development of this project was granted by the São Paulo Foundation for Research Support (FAPESP) and the National Council for Scientific and Technological Development (CNPq). The authors are grateful to Usina Santé S.A. for providing the samples, especially to Mr. Rodrigo Vezzani Franzé. We also thank the Centralized Multiuser Laboratory for Large Scale DNA Sequencing and Gene Expression Analysis (LMSeq) for the sequencing service (grant: 2009/53984-2).

\section{Conflict of interest}

The authors declare no conflict of interest regarding the publication of this paper.

\section{Author contributions}

WPO, EGML and JAMS conceived and designed the study; WPO, CCF, GCF and ESGP conducted the sampling and DNA sequencing; WPO, DGP, LTK and CDP conducted the bioinformatics analysis and analyzed the data and WPO and JAMS wrote the manuscript. All authors read and approved the final version.

\section{References}

Altschul SF, Gish W, Miller W, Myers EW and Lipman DJ (1990) Basic local alignment search tool. J Mol Biol 215:403-410. 
Aziz RK, Bartels D, Best AA, DeJongh M, Disz T, Edwards RA, Formsma K, Gerdes S, Glass EM, Kubal M et al. (2008) The RAST server: Rapid Annotations using Subsystems Technology. BMC Genomics 9:75.

Beisel CL and Afroz T (2016) Rethinking the hierarchy of sugar utilization in Bacteria. J Bacteriol 198:374-376.

Cragg SM, Beckham GT, Bruce NC, Bugg TD, Distel DL, Dupree P, Etxabe AG, Goodell BS, Jellison J, McGeehan JE et al. (2015) Lignocellulose degradation mechanisms across the Tree of Life. Curr Opin Chem Biol 29:108-119.

Fravet PRF, Soares RAB, Lana RMQ, Lana AMQ and Korndörfer GH (2010) Efeito de doses de torta de filtro e modo de aplicação sobre a produtividade e qualidade tecnológica da soqueira de cana-de-açúcar. Ciênc Agrotec 34:618-624.

Vries P and Visser J (2001) Aspergillus enzyme involved in degradation of plant cell wall polysaccharides. Microbiol Mol Biol 65:497-522.

Gopinath SCB, Anbu P, Md Arshad MK, Lakshmipriya T, Voon CH, Hashim U and Chinni SV (2017) Biotechnological processes in microbial amylase production. Biomed Res Int 2017:9.

Jourda C, Santini S, Rocher C, Le Bivic A and Claverie JM (2015) Draft genome sequence of an alphaproteobacterium associated with the Mediterranean sponge Oscarella lobularis. Genome Announc 3:e00977-15.

Katoh K and Standley DM (2013) MAFFT multiple sequence alignment software version 7: Improvements in performance and usability. Mol Biol Evol 30:772-80.

Kishi LT, Jesus RB, Pavani CD, Lemos EGM and Souza JAM (2015) Metagenomic assembly and draft genome sequence of an uncharacterized Prevotella sp. from Nelore rumen. Genome Announc 3:e00723-15.

Klein-Marcuschamer D, Oleskowicz-Popiel P, Simmons BA and Blanch HW (2012) The challenge of enzyme cost in the production of lignocellulosic biofuels. Bioresour Bioprocess 109:1083-1087.

Kroppenstedt RM and Goodfellow M (2006) The family Thermomonosporaceae: Actinocorallia, Actinomadura, Spirillospora and Thermomonospora. In: Dworkin M, Falkow S, Schleifer KH, Stackebrandt E (eds) The prokaryotes. Archaea and Bacteria: Firmicutes, Actinomycetes. 3rd edition. Springer, New York, vol 3, pp 682-724

Martins LF, Antunes LP, Pascon RC, Oliveira JCF, Digiampietri LA, Barbosa D, Peixoto BM, Vallim MA, Viana-Niero C, Ostroski EH et al. (2013) Metagenomic analysis of a tropical composting operation at the São Paulo Zoo Park reveals diversity of biomass degradation functions and organisms. PLoS One 8:e61928.

Richter M, Rosselló-Móra R, Glöckner FO and Peplies J (2016) JSpeciesWS: A web server for prokaryotic species circumscription based on pairwise genome comparison. Bioinformatics 32:929-931.

Trifinopoulos J, Nguyen LT, Von Haeseler A and Minh BQ (2016) W-IQ-TREE: A fast online phylogenetic tool for maximum likelihood analysis. Nucleic Acids Res 44:W232-W235.

Wang C, Dong D, Wang H, Müller K, Qin Y, Wang H and Wu W (2016) Metagenomic analysis of microbial consortia enriched from compost: new insights into the role of Actino- bacteria in lignocellulose decomposition. Biotechnol Biofuels 9:22.

Wang Y, Coleman-Derr D, Chen G and Gu YQ (2015) OrthoVenn: A web server for genome wide comparison and annotation of orthologous clusters across multiple species. Nucleic Acids Res 43:W78-W84.

Wei R, Oeser T, Then J, Kühn N, Barth M, Schmidt J and Zimmermann W (2014) Functional characterization and structural modeling of synthetic polyester-degrading hydrolases from Thermomonospora curvata. AMB Express 4:44.

Yin Y, Mao X, Yang JC, Chen X, Mao F and Xu Y (2012) dbCAN: A web resource for automated carbohydrate-active enzyme annotation. Nucleic Acids Res 40:W445-51.

\section{Internet resources}

Maddison WP and Maddison DR (2017) Mesquite: a modular system for evolutionary analysis. Version 3.2 http://mesquiteproject.org

Scythe v.0.991, https://github.com/vsbuffalo/scythe (November 24, 2017)

Figtree v.1.4.1, http://tree.bio.ed.ac.uk/software/figtree/ (November 24, 2017)

On-line Carbohydrate-Active enZYmes Database (CAZy), http://www.cazy.org/ (November 24, 2017).

On-line National Center for Biotechnology Information (NCBI), https://www.ncbi.nlm.nih.gov/ (November 24, 2017).

On-line DataBase for automated Carbohydrate-active enzyme Annotation (dbCAN), http://csbl.bmb.uga.edu/dbCAN/ (November 24, 2017).

On-line JSpecies Web Server (JSpeciesWS) v.3.0.11, http://jspecies.ribohost.com/jspeciesws/ (November 24, 2017).

On-line OrthoVenn, http://www.bioinfogenome.net/OrthoVenn (November 24, 2017).

On-line IQ-TREE v.1.5.5, http://iqtree.cibiv.univie.ac.at/ (November 24, 2017).

\section{Supplementary material}

The following materials are available online for this article: Table S1 - Scaffolds unspecific for Thermomonospora sp. identified with blastn in the draft genome CIT 1 dataset after separation of genomic clusters with Maxbin2.

Table S2 - Comparison between the partial draft genome CIT 1 statistics and strain DSM 43183 using Quast v.4.61.

Table S3 - Identifications of enzymes with activity on carbohydrate structures present in the draft genome CIT 1 recovered from metagenomic sequencing of filter cake.

Table S4 - Identifications of enzymes with activity on carbohydrate structures present in the circular genome of Thermomonospora curvata DSM 43183 (Thermomonosporaceae).

\section{Associate Editor: Juan Lucas Argueso Almeida}

License information: This is an open-access article distributed under the terms of the Creative Commons Attribution License (type CC-BY), which permits unrestricted use, distribution and reproduction in any medium, provided the original article is properly cited. 